\title{
A CRITERION OF OSCILLATION FOR GENERALIZED DIFFERENTIAL EQUATIONS*
}

\author{
WILLIAM T. REID
}

1. Introduction. For a second order ordinary linear differential equation

$$
\left[r(t) u^{\prime}(t)\right]^{\prime}+q(t) u(t)=0,
$$

with real-valued coefficient functions $r, q$ continuous and $r(t)$ positive on a non-compact interval $I=[a, \infty)$ the well-known WintnerLeighton criterion, (see Wintner [12], and Leighton [4]), states that (1.1) is oscillatory on arbitrary subintervals $[c, \infty)$ of $I$ whenever

$$
\int_{a}^{\infty} \frac{d s}{r(s)}=\lim _{t \rightarrow \infty} \int_{a}^{t} \frac{d s}{r(s)}=\infty,
$$

and

$$
\int_{a}^{\infty} q(s) d s=\lim _{r \rightarrow \infty} \int_{a}^{t} q(s) d s=\infty .
$$

The present paper presents an extension of this criterion for generalized matrix differential equations of the type previously considered by the author [6,9]. In particular, this extended criterion implies for self-adjoint systems of difference equations a result, which in the case of a scalar self-adjoint difference equation yields a generalization of a theorem on oscillation established by McCarthy [5], and answers a question raised by that author.

Matrix notation is used throughout; in particular, matrices of one column are called vectors, and for a vector $\left(y_{\alpha}\right),(\alpha=1, \cdots, n)$, the norm $|y|$ is given by $\left(\left|y_{1}\right|^{2}+\cdots+\left|y_{n}\right|^{2}\right)^{1 / 2}$. The $n \times n$ identity matrix is denoted by $E_{n}$, or merely by $E$ when there is no ambiguity, and 0 is used indiscriminately for the zero matrix of any dimensions; the conjugate transpose of a matrix $M$ is designated by $M^{*}$. The relations $M \geqq N,(M>N)$, are used to signify that $M$ and $N$ are hermitian matrices of the same dimensions and $M-N$ is a non-negative, (positive), definite matrix. A matrix function is called continuous,

Received by the editors on October 1, 1976.

*This research was supported by the National Science Foundation under Grant MPS74-15648. 
integrable, absolutely continuous, etc., when each element of the matrix possesses the specified property. If a matrix function $M(t)$ is locally absolutely continuous on an interval $I$, then $M^{\prime}(t)$ signifies the matrix of derivatives at values where these derivatives exist, and zero elsewhere. Similarly, if $\boldsymbol{M}(t)$ is Lebesgue integrable on a compact subinterval $[a, b]$ of $I$ then $\int_{a}^{b} M(t) d t$ denotes the matrix of integrals of respective elements of $M(t)$. If $M(t)$ and $N(t)$ are matrix functions which have a common domain of existence, and $M(t)$ and $N(t)$ are equal almost everywhere (Lebesgue) on this domain, we write simply $M(t)=N(t)$.

2. The basic oscillation theorem. Consider a generalized vector differential system

$$
-d v(t)-[d \mathrm{~S}(t)] u(t)=0, u^{\prime}(t)-B(t) v(t)=0,
$$

in $n$-dimensional vector functions $u, v$ with coefficient matrix functions satisfying the following hypothesis on a given non-compact interval $I=[a, b)$, where $-\infty<a<b \leqq \infty$ :

(פ) $B$ and $S$ are hermitian $n \times n$ matrix functions, with $B$ locally of class $\mathcal{L}^{\infty}$ and $S$ locally of bounded variation, while $B(t) \geqq 0$ for $t \in I$.

For basic properties of such systems, and the relation of such systems to ordinary differential systems to which (2.1) reduces when $S(t)$ is locally absolutely continuous, the reader is referred to [6] and [9] of the Bibliography. In particular, two values $t_{1}$ and $t_{2}$ of $I$ are called (mutually) conjugate with respect to (2.1) if there exists a solution $(u ; v)$ of this system with $u \neq 0$ on the subinterval with endpoints $t_{1}$ and $t_{2}$, while $u\left(t_{1}\right)=0=u\left(t_{2}\right)$. Such a system is said to be disconjugate on a subinterval $I_{0}$ of $I$ provided no two distinct points of $I_{0}$ are conjugate. On the non-compact interval $I=[a, b)$ the system is said to be oscillatory near $b$, (oscillatory for large $t$ if $b=\infty$ ), in case (2.1) is not disconjugate on an arbitrary non-degenerate subinterval $[c, b)$ of $I$.

In view of the assumption that $B(t) \geqq 0$ and $B$ is locally of class $\mathcal{L}^{\infty}$ on $I$, the smallest eigenvalue $\lambda_{\text {Min }}[B(t)]$ of $B(t)$ is a nonnegative, real-valued function that is locally of class $\mathcal{L}^{\infty}$ on $I$, (see, for example, [10; Theorem 3.1]). The basic oscillation result to be established is as follows.

THEOREM 2.1. If hypothesis ( $\mathfrak{W})$ holds, then (2.1) is oscillatory near $b$ whenever the following two conditions are satisfied: 
(i) $\lambda(t)=\lambda_{\operatorname{Min}}[B(t)]$ is such that $\int_{a}^{b} \lambda(s) d s=$ $\lim _{t \rightarrow b} \int_{a}^{t} \lambda(s) d s=\infty$;

(ii) there exists an $n$-dimensional vector $\xi$ such that $|\xi|=1$, and $\xi^{*} \mathrm{~S}(t) \xi \rightarrow \infty$ as $t \rightarrow b$.

For $r, q$ continuous real-valued functions on $[a, \infty)$ with $r$ positive, the result of the above theorem for $n=1, B(t)=1 / r(t)$, and $S(t)=$ $\int_{a}^{t} q(s) d s$, reduces to the Wintner-Leighton criterion for the scalar ordinary differential equation (1.1).

If hypothesis $(\mathfrak{g})$ holds, and there is a subinterval $(c, b)$ on which (2.1) is disconjugate, then there exists an hermitian matrix function $N(t)$ and an hermitian constant matrix $\chi$ such that for $s \in(c, b)$,

$$
N(t)-\int_{s}^{t} N(r) B(r) N(r) d r=S(t)+\chi, t \in(c, b) .
$$

This result is of the form of conclusion (iii) of Theorem 5.1 of [9], with the substitutions $N(t)=-W(t), S(t)=-M(t), \chi=-\Psi$, but is not a direct consequence of the cited result of [9] as the latter deals with a compact interval. The stated result may be proved by the same method as that presented for conclusion (iii) of Theorem 5.1 of [9], however, with the modification that one now refers to Theorem 5.3 of the author's earlier paper [8], rather than to Theorem 5.1 of [8]. In this connection, it is to be emphasized that the results of $\$ 5$ of [8] do not involve any assumption of normality on subintervals, so that for the existence of $N(t)$ and $\chi$ satisfying (2.3) no such assumption is needed.

Now if $|\xi|=1$, and $\xi^{*} S(t) \xi \rightarrow \infty$ as $t \rightarrow b$, then also $\xi^{*}[S(t)+$ $\chi] \xi \rightarrow \infty$ as $t \rightarrow b$, and as $B(t) \geqq 0$ on $I$ equation (2.3) implies that $\xi^{*} N(t) \xi \rightarrow \infty$ as $t \rightarrow b$. Also, since $N(t)$ and $B(t)$ are hermitian we have $\xi^{*} N(r) B(r) N(r) \xi \geqq \lambda(r) \xi^{*} N^{2}(r) \xi$. Moreover, by the Schwarz inequality, it follows that $\xi^{*} N^{2}(r) \xi=\left[\xi^{*} \xi\right]\left[\xi^{*} N^{2}(r) \xi\right] \geqq\left|\xi^{*} N(r) \xi\right|^{2}$. Consequently, whenever conditions (2.2) are satisfied, and $N(t), \chi$ are as in (2.3), there exists a value $s \in(c, b)$ such that $\theta(t)=\xi^{*} N(t) \xi$ satisfies the conditions

$$
\begin{aligned}
& \text { (i) } \theta(t)>0 \text { for } t \in[s, b), \\
& \text { (ii) } \theta(t)-\int_{s}^{t} \lambda(r) \theta^{2}(r) d r \rightarrow \infty \text { as } t \rightarrow b \text {. }
\end{aligned}
$$

However, these conditions are incompatible with the assumption that $\int_{s}^{t} \lambda(r) d r \rightarrow \infty$ as $t \rightarrow b$. Indeed, if $M>0$ and $\tau \in(s, b)$ is such that $\theta(t)-\int_{s}^{t} \lambda(r) \theta^{2}(r) d r \geqq M$ for $t \in[\tau, b)$, then

$$
\lambda(t) \theta^{2}(t)\left[M+\int_{\tau}^{t} \lambda(r) \theta^{2}(r) d r\right]^{-2} \geqq \lambda(t), \text { for } t \in[\tau, b),
$$


and integration yields the inequality $M^{-1} \geqq \int_{\tau}^{t} \lambda(r) d r$, for $t \in[\tau, b)$, contrary to $(2.2 \mathrm{i})$. Consequently, whenever $(\mathfrak{H})$ and conditions (2.2i, ii) hold the system (2.1) fails to be disconjugate on subintervals of $I$ of the form $(c, b)$; that is, $(2.1)$ is oscillatory near $b$.

If (2.1) is identically normal, and $S(t)$ is locally absolutely continuous with derivative $S^{\prime}(t)=C(t)$ almost everywhere on $I$, then in case $C(t) \geqq 0$ on a subinterval $(c, b)$ of $I$ it follows from Theorem 3.3 of [7] that the conclusion of Theorem 2.1 remains valid when (2.2i) is replaced by the weaker condition

$\left(2.5_{0}\right) \xi^{*}\left[\int_{a}^{t} B(s) d s\right] \xi \rightarrow \infty$ as $t \rightarrow b$, for arbitrary non-zero vectors $\xi$, which, in view of the non-negative definite character of $B(t)$, is equivalent to the condition

$$
\lambda_{\text {Min }}\left[\int_{a}^{t} B(s) d s\right] \rightarrow \infty \text { as } t \rightarrow b .
$$

For a discussion of various related criteria, the reader is referred to the papers $[1,2]$ of Ahlbrandt. It is to be remarked, however, that it is not known whether or not the result of Theorem 2.1 remains valid whenever $(2.2 \mathrm{i})$ is replaced by (2.5) and no additional conditions are imposed.

3. Criteria for related systems. Now consider a self-adjoint generalized differential system of the form

$$
-d v_{1}(t)+\left[C_{1}(t) u_{1}(t)\right.
$$

$$
\begin{aligned}
& \left.-A_{1}{ }^{*}(t) v_{1}(t)\right] d t-\left[d S_{1}(t)\right] u_{1}(t)=0, \\
& u_{1}{ }^{\prime}(t)-A_{1}(t) u_{1}(t)-B_{1}(t) v_{1}(t)=0,
\end{aligned}
$$

wherein the $n \times n$ matrix functions $A_{1}, B_{1}, C_{1}, S_{1}$ satisfy on $I=$ $[a, b)$ the following hypothesis.

$\left(\mathfrak{S}_{1}\right) A_{1}, B_{1}, C_{1}$ are locally of class $\mathcal{L}^{\infty}$, with $B_{1}, C_{1}$ hermitian and $B_{1}(t) \geqq 0$ almost everywhere on $I$, while $S_{1}$ is locally of bounded variation on this interval.

A system (3.1) is reducible to the form (2.1) under various substitutions. In particular, if $Y_{1}(t)$ is a fundamental matrix solution of the ordinary differential equation $Y_{1}{ }^{\prime}(t)-A_{1}(t) Y_{1}(t)=0$, then under the substitution

$$
u_{1}(t)=Y_{1}(t) u(t), v_{1}(t)=Y_{1}{ }^{*-1}(t) v(t),
$$


the system (3.1) is reduced to $(2.1)$ with

$$
\begin{aligned}
& B(t)=Y_{1}^{-1}(t) B_{1}(t) Y_{1}^{*-1}(t) \\
& S(t)=\int_{t_{0}}^{t} Y_{1}^{*}(s)\left[d S_{1}(s)\right] Y_{1}(s)-\int_{t_{0}}^{t} Y_{1}^{*}(s) C(s) Y_{1}(s) d s
\end{aligned}
$$

(see, for example, $[9, \S 2]$ ).

For a system (3.1) with

$$
S_{1}(t)=M(t)+M_{1}(t),
$$

where $M$ and $M_{1}$ are hermitian matrix functions that are locally of bounded variation on $I$, and the system

$$
\begin{aligned}
-d v_{1}(t)+\left[C_{1}(t)\right. & u_{1}(t) \\
- & \left.A_{1}{ }^{*}(t) v_{1}(t)\right] d t-\left[d M_{1}(t)\right] u_{1}(t)=0, \\
& u_{1}{ }^{\prime}(t)-A_{1}(t) u_{1}(t)-B_{1}(t) v_{1}(t)=0,
\end{aligned}
$$

is disconjugate on a subinterval $(c, b)$ of $I$, let $\left(U_{1}(t) ; V_{1}(t)\right)$ be a conjoined basis of $(3.5)$ with $U_{1}(t)$ non-singular on $(c, b)$. Under the substitution

$$
u_{1}(t)=U_{1}(t) u(t), v_{1}(t)=V_{1}(t) u(t)+U_{1}^{*-1}(t) v(t),
$$

the system (3.1) reduces on $(c, b)$ to the form $(2.1)$ with

$$
B(t)=U_{1}^{-1}(t) B_{1}(t) U_{1}^{*-1}(t), S(t)=\int_{t_{0}}^{t} U_{1}^{*}(s)[d M(s)] U_{1}(s) .
$$

For hermitian ordinary differential systems appearing as the accessory system for variational problems of Lagrange or Bolza type this transformation is essentially the classical Clebsch transformation, (see [11, Lemma 4.2 and Corollary of Chapter VII], and also [9, Lemma 5.1]).

Moreover, in view of the equivalence of (3.5) to an ordinary differential system as in $[9, \S 2]$, and the results on principal solutions of self-adjoint ordinary differential systems presented in $[11, \S 3$ of Chapter VII], it follows that if (3.5) is identically normal on $I$ then $B(t)$ defined by (3.7) is such that for $c<s<t<b$ the hermitian matrix function $\Theta\left(t, s \mid U_{1}\right)=\int_{s}^{t} B(r) d r$ is positive definite and $\Theta^{-1}\left(t, s \mid U_{1}\right) \rightarrow 0$ as $t \rightarrow b$, which is equivalent to $\left(2.5_{0}\right)$ or $(2.5)$.

4. Self-adjoint systems of difference equations. Now for a given non-compact interval $I=[a, b)$ let $\left\{t_{j}\right\},(j=0,1, \cdots)$, be a sequence of values satisfying 


$$
a=t_{0}<t_{1}<\cdots<t_{j}<t_{j+1}<\cdots, \lim _{j \rightarrow \infty} t_{j}=b,
$$

and let $R_{j}, S_{j},(j=0,1, \cdots)$, be hermitian $n \times n$ matrices with each $R_{j}$ positive definite. Consider a system $(2.1)$ wherein for $j=0,1, \cdots$ we have

$$
B(t) \equiv R_{j}^{-1}, S(t) \equiv S_{j}, \text { for } t \in\left(t_{j}, t_{j+1}\right) .
$$

For definiteness, one may also suppose

$$
S\left(t_{j+1}\right)=\frac{1}{2}\left[S_{j}+S_{j+1}\right],(j=0,1, \cdots) .
$$

As presented in $[6, \S 6]$, if $(u ; v)$ is a solution of $(2.1)$ on $I$ then on this interval $u(t)$ is a continuous vector function that is linear on each subinterval $\left[t_{j}, t_{j+1}\right]$, and for

$$
\begin{gathered}
K_{j+1}=S_{j+1}-S_{j}, \Delta u\left(t_{j}\right)=u\left(t_{j+1}\right)-u\left(t_{j}\right), \\
\Delta t_{j}=t_{j+1}-t_{j}
\end{gathered}
$$

the sequence $\left\{u\left(t_{j}\right)\right\}$ satisfies the self-adjoint system of difference equations

$$
R_{j+1} \frac{\Delta u\left(t_{j+1}\right)}{\Delta t_{j+1}}-R_{j} \frac{\Delta u\left(t_{j}\right)}{\Delta t_{j}}+K_{j+1} u\left(t_{j+1}\right)=0,(j=0,1, \cdots)
$$

Conversely, if on $I$ the vector function $u(t)$ is continuous, linear on each subinterval $\left[t_{j}, t_{j+1}\right]$, and satisfies (4.4), then a solution of $(2.1)$ is given by $u=u(t), v=v(t)$ with

$$
\begin{aligned}
v\left(t_{0}\right) & =R_{0} \frac{\Delta u\left(t_{0}\right)}{\Delta t_{0}}-\left[S\left(t_{0}\right)-S_{0}\right] u\left(t_{0}\right), \\
v(t) & =R_{j} \frac{\Delta u\left(t_{j}\right)}{\Delta t_{j}} \text { for } t \in\left(t_{j}, t_{j+1}\right), \\
v\left(t_{j+1}\right) & =R_{j} \frac{\Delta u\left(t_{j}\right)}{\Delta t_{j}}-\left[S\left(t_{j+1}\right)-S_{j}\right] u\left(t_{j+1}\right),(j=0,1, \cdots) .
\end{aligned}
$$

For a direct treatment of real self-adjoint systems (4.4), with the derivation of certain central oscillation and comparison theorems, the reader is referred to Harris [3].

Since for $t \in\left(t_{j}, t_{j+1}\right)$ we have $\lambda_{\operatorname{Min}}[B(t)]=1 / \lambda_{\operatorname{Max}}\left[R_{j}\right]$, a direct application of Theorem 2.1 .yields the following result.

TheOREM 4.1. If $I=[a, b)$, and $t_{j}, R_{j}, S_{j},(j=0,1, \cdots)$, are as specified above, then (4.4) is oscillatory near $b$ when the following conditions are satisfied: 
(i) $\sum_{j=0}^{\infty}\left(\Delta t_{j}\right) / \lambda_{\operatorname{Max}}\left[R_{j}\right]=\infty$,

(ii) there exists a vector $\xi$ with $|\xi|=1$ and $\sum_{j=0}^{\infty} \xi^{*} K_{j+1} \xi$ $=\lim _{m \rightarrow \infty} \Sigma_{j=1}^{m} \xi^{*} K_{j+1} \xi=\infty$.

For $n=1, a=0, b=\infty, t_{j}=j,(j=0,1, \cdots)$, and $r_{j}, k_{j}$ real values with each $r_{j}$ positive, this criterion states that the real self-adjoint difference equation

$$
r_{j+1} \Delta u\left(t_{j+1}\right)-r_{j} \Delta u\left(t_{j}\right)+k_{j+1} u\left(t_{j+1}\right)=0,(j=0,1, \cdots),
$$

is oscillatory near $\infty$ whenever

$$
\begin{array}{ll}
\text { (i) } \sum_{j=0}^{\infty} 1 / r_{j}=\infty, & \text { (ii) } \sum_{j=0}^{\infty} k_{j+1}=\infty .
\end{array}
$$

This condition generalizes the result of McCarthy [5], whose criterion for the oscillation of (4.5) near infinity involved (4.6ii) and the boundedness of the positive sequence $\left\{r_{j}\right\}$, and who stated $[5, \mathrm{p}$. 204 that he did not know whether the boundedness of $\left\{r_{j}\right\}$ could be replaced by the divergence of the series $\sum_{j=0}^{\infty} 1 / r_{j}$.

Added in proof. Subsequent to the submission of the present paper the author received from Don B. Hilton and Roger T. Lewis a copy of a manuscript entitled Spectral Analysis of Order Difference Equations, wherein they also obtain the above stated generalization of the result of McCarthy [5].

\section{Bibliography}

1. C. D. Ahlbrandt, Disconjugacy criteria for self-adjoint differential systems, (Dissertation, University of Oklahoma, 1968), Journal of Differential Equations 6 (1969), 271-295. MR 39 \#3555.

2. - The question of equivalence of principal and co-principal solutions of self-adjoint differential systems, Illinois Journal of Mathematics 20 (1976), 72-81. MR 53 \#940.

3. V. C. Harris, A system of difference equations and an associated boundary value problem, (Dissertation, Northwestern University, 1950).

4. W. Leighton, On self-adjoint differential equations of the second order, Journal of the London Mathematical Society 27 (1952), 37-47. MR 13-745.

5. P. J. McCarthy, Note on the oscillation of solutions of second order difference equations, Portugaliae Mathematica 18 (1959), 203-205. MR 23 \#A1953.

6. W. T. Reid, Generalized linear differential equations, Journal of Mathematics and Mechanics 8 (1959), 705-726. MR 21 \#5777.

7. - Oscillation criteria for self-adjoint differential systems, Transactions of the American Mathematical Society 101 (1961), 91-106. MR 24 \#A3349.

8. - Principal solutions of nonoscillatory linear differential systems, Journal of Mathematical Analysis and Applications 9 (1964), 397-423. MR 29 \#6110. 
9. —_ Generalized linear differential systems and related Riccati matrix integral equations, Illinois Journal of Mathematics 10 (1966), 701-722. MR 37 \# 1682.

10. - Some elementary properties of proper values and proper vectors of matrix functions, SIAM Journal of Applied Mathematics 18 (1970), 259-266. MR 41 \#8623.

11. _ Ordinary Differential Equations, John Wiley and Sons, New York, 1971. MR 42 \#7963.

12. A. Wintner, A criterion of oscillatory stability, Quarterly of Applied Mathematics 7 (1949), 115-117. MR 10-456.

Department of Mathematics, University of Oklahoma, Norman, Oklahoma 73019

Present address: Department of Mathematics, University of Texas at Austin, Austin, Texas 78712 